\title{
A relation between algebraic and transform-based reconstruction technique in computed tomography
}

\author{
S. Kiefhaber, M. Rosenbaum, W. Sauer-Greff, and R. Urbansky \\ Technical University of Kaiserslautern, Chair of Communications Engineering, Germany \\ Correspondence to: S. Kiefhaber (s.kiefhaber@t-online.de)
}

\begin{abstract}
In this contribution a coherent relation between the algebraic and the transform-based reconstruction technique for computed tomography is introduced using the mathematical means of two-dimensional signal processing. There are two advantages arising from that approach. First, the algebraic reconstruction technique can now be used efficiently regarding memory usage without considerations concerning the handling of large sparse matrices. Second, the relation grants a more intuitive understanding as to the convergence characteristics of the iterative method. Besides the gain in theoretical insight these advantages offer new possibilities for application-specific fine tuning of reconstruction techniques.
\end{abstract}

\section{Introduction}

Computed tomography is a well-established method in medicine, material science and quality control. It is an imaging technique used to create a cross-sectional image of the interior of a body to be examined. In contrast to classical imaging where the image corresponds directly to the measurements, in computed tomography the image is generated in an indirect manner. Therefore a series of transmission measurements, e.g. utilising x-rays, is taken. The projection data can be used to reconstruct an image of the inner structure of an object.

Concerning algorithms, the key part of every tomographic imaging system is the reconstruction technique. There are two major reconstruction techniques that are considered in this paper. The first, transform-based approach, was introduced in 1917 by the Austrian mathematician Johann Radon (Radon, 1917). The Radon transform and its inverse are analytical expressions of the projection process during the transmission measurements and the reconstruction technique, respectively. They are well understood from a signal process- ing point of view. The second, algebraic reconstruction technique is attributed to Bender et al. and, in principal, is an accomplishment of the Polish mathematician Stefan Kaczmarz who proposed an iterative method to approximate solutions of systems of linear equations (Kaczmarz, 1937). In terms of linear algebra this technique is well known.

The main contribution of this paper is the reinterpretation of the algebraic reconstruction technique by means of twodimensional signal processing. Starting with the analysis and reinterpretation of the description of the measurement process using a system of linear equations in Subsect. 3.1 this leads to a mathematical relation between the algebraic and the transform-based reconstruction technique.

Knowledge about this relation has two advantages. First, the algebraic reconstruction technique can now be implemented efficiently as to the usage of memory avoiding the necessity to handle large sparse matrices. Second, the closed form of the iteration exactly describes the convergence characteristics of the iterative method. In addition to the academical benefit these advantages could offer new possibilities for application-specific fine tuning of reconstruction techniques.

The remainder of this publication is organized as follows. A short introduction to the process of data acquisition in computed tomography along with the mathematical description of the measured data, namely the Radon transform, is given in Subsect. 2.1. The essential facts about the inverse Radon transform are revisited in Subsect. 2.2 in a intuitive manner based on Morneburg (1995) avoiding the indeed exact but also cumbersome mathematical derivation. The principal ideas of Kaczmarz' method are summarised in Subsect. 2.3. The reinterpretation of the algebraic reconstruction technique is presented in Sect. 3. The proof of the relation between the two methods is given in Subsect. 3.2. The implications of the introduced relation between algebraic and transform-based reconstruction technique are discussed in 


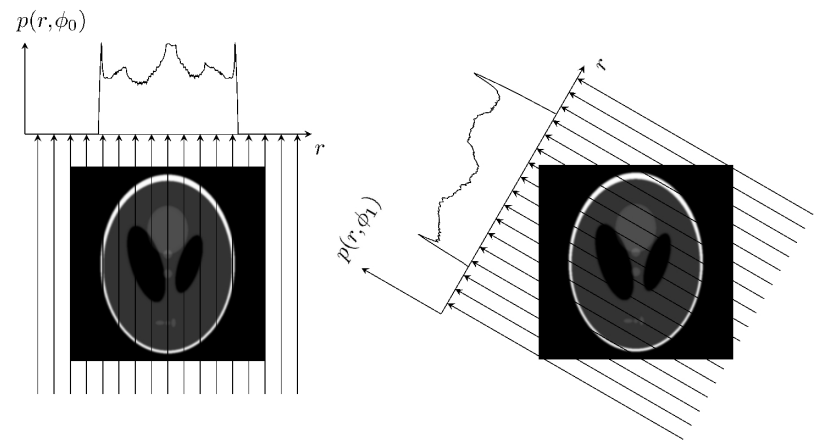

Fig. 1. Measurement of the attenuation of x-rays on their paths along parallels through the phantom repeatedly for different directions, here exemplary for two different angles.

Subsect. 3.4. A concluding summary of the basic ideas and insights of this contribution is given in Sect. 4 .

\section{Preliminary Considerations}

In this section a short recapitulation of the underlying ideas and the necessary preliminary considerations concerning computed tomography, the transform-based and the algebraic reconstruction technique are given.

\subsection{Computed tomography}

Computed tomography was first successfully conducted by Sir Godfrey Newbold Hounsfield in 1971 who in 1979 received the Nobel Price in Physiology or Medicine for his efforts together with Allan McLeod Cormack. With his device he measured the accumulated attenuation of $\mathrm{x}$-rays on their paths along parallels through the phantom repeatedly for different directions of equally distributed angles between zero and 180 degrees. This is illustrated in Fig. 1.

Mathematically the measured attenuation can be expressed as the Radon transform of the function describing the spacial distribution of the attenuation coefficient. The local attenuation coefficient in a plane $(x, y) \in \mathbb{R}^{2}$ is expressed as a continuous function $g(x, y)$. The paths are expressed as straight lines $G(r, \phi):\{(x, y) \mid 0=r-x \cos (\phi)-y \sin (\phi)\}$ where $r$ is the perpendicular distance of $G$ to the origin and $\phi$ is the angle between $G$ and the y-axis. The ensemble of integrals over $g(x, y)$ along the paths defined by $G(r, \phi)$ is the Radon transform

$p(r, \phi)=\mathcal{R}\{g(x, y)\}=\int_{G(r, \phi)} g(x, y) \mathrm{d} x \mathrm{~d} y$

and corresponds to the measured data. The main goal in computed tomography is to reconstruct the function describing the original spacial distribution of the attenuation coefficient $g(x, y)$ from the integral values measured along parallel

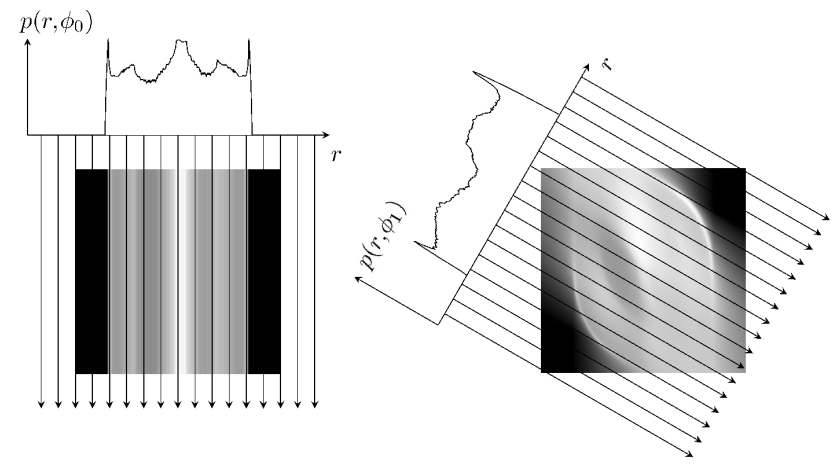

Fig. 2. Illustration of the backprojection operation.
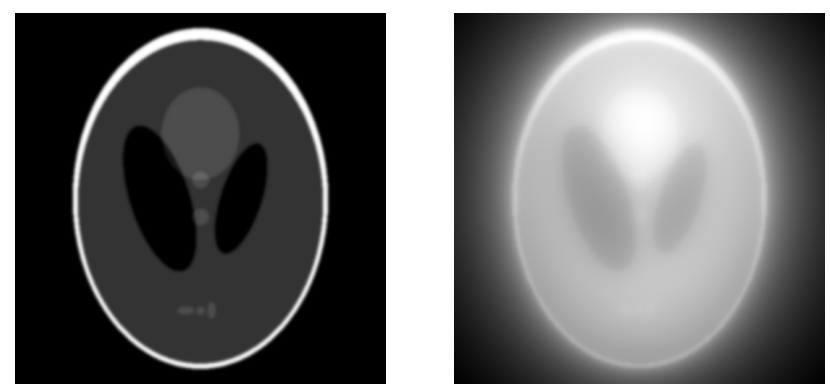

Fig. 3. Comparison of original and result of plain backprojection.

paths from different directions as represented by the Radon transform $p(r, \phi)$. The practical means to solve this problem is given by so-called reconstruction techniques.

For further details on the Radon transform as well as its inverse which is subject to Subsect. 2.2 please cf. Cho et al. (1993); Morneburg (1995); Poularikas (1996).

\subsection{Transform-based reconstruction technique}

Having the Radon transform in mind, the first idea to solve the reconstruction problem is quite manifest, namely inversion of the measurement process by backprojecting the integral values onto their respective paths and accumulating the portions from each direction. Mathematically, this backprojection can be written as the integral of $p(r, \phi)$ over $\phi$.

$\mathcal{B}\{p(r, \phi)\}=\int_{0}^{\pi} p(r, \phi) \mathrm{d} \phi$

The backprojection operation is illustrated in Fig. 2. Figure 3 shows the original image as well as the result of the consecutive Radon transform and backprojection. Obviously, the reconstruction resulting from the plain backprojection differs significantly from the original image by a certain blurring effect. 


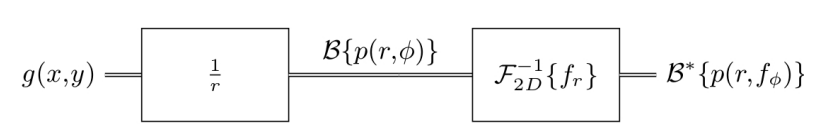

Fig. 4. 2-D transmission system consisting of Radon transform, backprojection and compensating filter.

The next step towards the solution of the reconstruction problem is to treat the concatenation of Radon transform and backprojection as a two-dimensional linear transmission system. Thereby it is possible to identify the transmission behaviour of the system and compensate for it by adding a filter with the inverse behaviour to the signal processing chain.

According to Deans (1983), the impulse response as well as the transfer function of the linear transmission system consisting of the concatenation of Radon transform and backprojection decay according to the reciprocal radial distance from the origin, i.e. $h_{\mathcal{R B}}=\frac{1}{r}$ and $H_{\mathcal{R B}}=\frac{1}{f_{r}}$ in the original and the Fourier domain, respectively. The functional identity of the impulse response $h_{\mathcal{R} \mathcal{B}}$ and the transfer function $H_{\mathcal{R} \mathcal{B}}$ is called a fix point of the Hankel transform of order zero which for rotationally symmetric functions is identical to the Fourier transform in two dimensions. The transfer function of the composite system $H_{\mathcal{R} \mathcal{B}}=\frac{1}{f_{r}}$ will play a key role in the derivation of the relation between the transform-based and the algebraic reconstruction technique.

The impulse response of the compensating filter follows from inverting the transfer function $H_{\mathcal{R} \mathcal{B}}$ and then taking its two-dimensional inverse Fourier transform. Mathematically, the filtering corresponds to a convolution which, incorporated into Eq. (2), leads to the so-called filtered backprojection.

$\mathcal{B}^{*}\{p(r, \phi)\}=\int_{0}^{\pi} p(r, \phi) * \mathcal{F}_{2 D}^{-1}\left\{f_{r}\right\} \mathrm{d} \phi$

The filtered backprojection is one representation of the inverse Radon transform and an analytical solution to the reconstruction problem. Discretization and implementation leads to the transform-based reconstruction technique. Figure 4 illustrates the described construction of the filtered backprojection. The inverse Radon transform can also be derived in a strictly mathematical manner as, e.g., shown in Poularikas (1996). The strictly mathematical derivation allows to identify the transfer function of the compensating filter $f_{r}$ as the determinant of the Jacobian matrix altered due to the transform from Cartesian to polar coordinates which is inherent to the geometry of the measurement process.

\subsection{Algebraic reconstruction technique}

In comparison to transform-based methods, the derivation of the algebraic reconstruction technique follows a somewhat

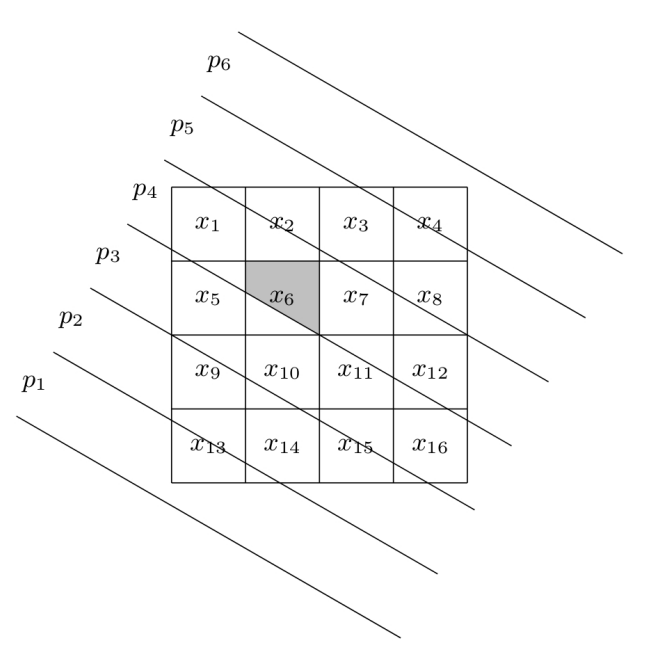

Fig. 5. Setting up the equation system by defining one linear equation per ray.

different approach. The reconstruction problem is stated directly in a discretised form rather than continuously as for the transform-based reconstruction technique. Figure 5 shows a four by four pixel area to be reconstructed which is overlayed with six corridors corresponding to the parallel paths for one exemplary direction. The commonly used approach (see, e.g., Kak et al., 2001) is to set up one linear equation for each path considering all directions

$x_{1} a_{1, j}+x_{2} a_{2, j}+\ldots+x_{N} a_{N, j}=p_{j}$

and to combine them to a system of linear equations written here using a vector matrix formalism.

$\mathbf{A x}=\mathbf{p}$

The individual coefficients $a_{i, j}$ may for example be chosen to account for the intersectional area of the pixel $i$ with corridor $j$. Also common practice is to use a hit-or-miss approach in order to reduce the computational effort. If a pixel intersects with the corridor at hand the respective coefficient is set to one, otherwise to zero.

In the linear equation system (5) vector $\mathbf{p}$ corresponds to the measured data, matrix $\mathbf{A}$ accounts for the geometry of the tomographic system and vector $\mathbf{x}$ corresponds to the wanted spacial distribution of the attenuation coefficient. On first sight the solution of the reconstruction problem is obtained by solving the system of linear equations for $\mathbf{x}$. However, there are certain difficulties that in general prohibit the straight forward solution of Eq. (5).

First, matrix A does not necessarily need to be square or, second, invertible at all for that matter. Even if $\mathbf{A}$ is a square matrix possible measurement noise might disrupt an exact solution. Third, the mere size of the equation system for any real tomographic system makes a solution, for example using 


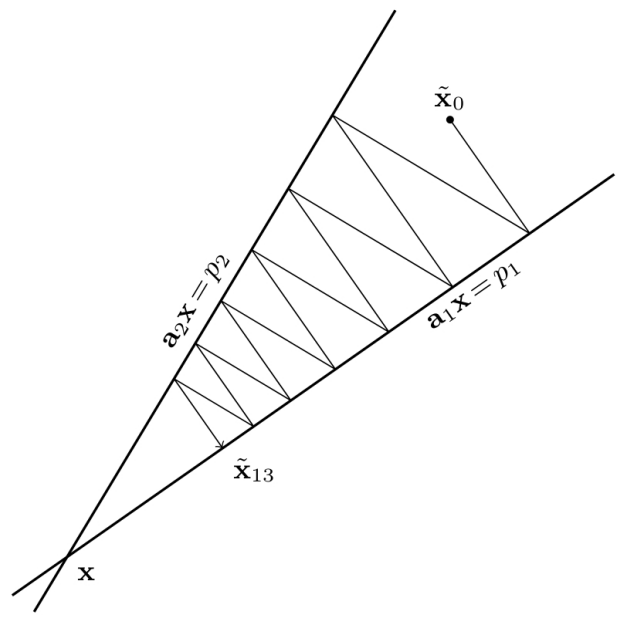

Fig. 6. Move arbitrary point $\tilde{\mathbf{x}}_{\mathbf{0}}$ towards the intersectional point of hyperplanes through successive orthogonal projections to find a solution of system of linear equations. Exemplary for first 13 iterations.

the Moore-Penrose pseudoinverse to approximate a solution, computationally extremely expensive.

Therefore it is advisable to utilize an iterative method to directly determine (in general) an approximation $\tilde{\mathbf{x}}$ to a solution $\mathbf{x}$ of the system of linear equations. In computed tomography there are several methods used such as the algebraic reconstruction technique as suggested by Bender et al., the simultaneous iterative reconstruction technique or the simultaneous algebraic reconstruction technique (cf. e.g. Kak et al., 2001). All of them are in principle based on a method proposed by (Kaczmarz, 1937).

His idea was to interpret $\mathbf{x}$ as a point in an $n$-dimensional space. Therewith every line of the linear equation system can be used to define a hyperplane in that space. The intersection of all hyperplanes, provided it exists, corresponds to the solution of the system of linear equations. So, to solve the system of linear equations means to find the intersectional point of all hyperplanes. Kaczmarz suggested to do so by choosing an arbitrary point $\tilde{\mathbf{x}}_{\mathbf{0}}$ in the n-dimensional space and moving the initially guessed point towards the real solution through an iterative process of successive orthogonal projections on the hyperplanes. This process is illustrated for a two-dimensional solution-space in Fig. 6.

Mathematically, Kaczmarz' method can be written as follows. For a detailed derivation cf., e.g., Kak et al. (2001).

$\tilde{\mathbf{x}}_{j}=\tilde{\mathbf{x}}_{j-1}+\frac{\mathbf{a}_{j}^{\top}}{\left|\mathbf{a}_{j}\right|}\left(p_{j}-\tilde{p}_{j}\right) \quad$ where $\quad \tilde{p}_{j}=\mathbf{a}_{j} \cdot \tilde{\mathbf{x}}_{j-1}$

Here $\tilde{\mathbf{x}}_{j}$ is the approximation to the solution of the system of linear equations after $j$ orthogonal projections, $\mathbf{a}_{j}$ is the $j$-th line of the coefficient matrix $\mathbf{A}$, and $p_{j}$ is the $j$-th measured value and $\tilde{p}_{j}$ is the result of the linear mapping defined in (5) assuming $\tilde{\mathbf{x}}_{j}$ is the wanted spacial distribution of the attenuation coefficient. According to Kaczmarz (1937); Tanabe (1971), if for every value of $n$ one full projection cycle is conducted it follows that in the limit $n$ to infinity $\tilde{\mathbf{x}}_{n}$ approximates the solution with arbitrary accuracy.

$\lim _{n \rightarrow \infty} \tilde{\mathbf{x}}_{n}=\mathbf{x}$

Note that if there is no unique solution, the estimate oscillates within the neighbourhood of the intersections.

\section{Relation of methods}

The first step to derive a relation between the inverse Radon transform and Kaczmarz' method is to reexamine the initial model on which the setup of the system of linear equations is based. This leads to a reinterpretation of Kaczmarz' method as iterative backprojection. Thereafter the transmission behaviour of the iterative backprojection will be closely examined by presenting a closed form representation which also exactly describes the convergence characteristics of the iterative method.

\subsection{Reinterpretation of linear mapping as discretised Radon transform}

The representations of the distribution function and the paths in terms of pixels and corridors, respectively, correspond to the usage of a Haar basis; in terms of sampling and interpolation theory it corresponds to interpolation with B-splines of order zero or, colloquial, to a nearest neighbour interpolation (cf., e.g., Unser, 2000). By disregarding the particular method of interpolation the setup of the linear equation system can be done assuming the elements of vectors $\mathbf{x}$ and $\mathbf{p}$ being the weights of a Dirac basis representations, i.e. the samples of the continuous function describing the spacial distribution of the attenuation coefficients and their directed sums as discretisation of the path integrals, respectively. This is illustrated in Fig. 7. The particular method of interpolation is accounted for by the values of the coefficient matrix $\mathbf{A}$.

From that point of view it becomes comprehensible that depending on the kind of interpolation the system of linear Eq. (5) is essentially a discretisation of the Radon transform. By applying this idea on Eq. (6) Kaczmarz' method can be understood as iterative backprojection of the difference between virtual projection data $\tilde{p}_{n}$ computed using the estimated distribution function $\tilde{\mathbf{x}}_{n-1}$ and the actually measured data $p$.

Based on the above considerations Eq. (6) can be rewritten using the Radon transform and backprojection operators on the continuously defined distribution function.

$$
\begin{aligned}
\tilde{g}_{n+1} & =\tilde{g}_{n}+\mathcal{B}\left(p-\mathcal{R} \tilde{g}_{n}\right) \quad \text { where } \quad p=\mathcal{R} g \\
& =\tilde{g}_{n}+\mathcal{B}\left(\mathcal{R} g-\mathcal{R} \tilde{g}_{n}\right) \\
& =\tilde{g}_{n}+\mathcal{B} \mathcal{R} g-\mathcal{B} \mathcal{R} \tilde{g}_{n}
\end{aligned}
$$




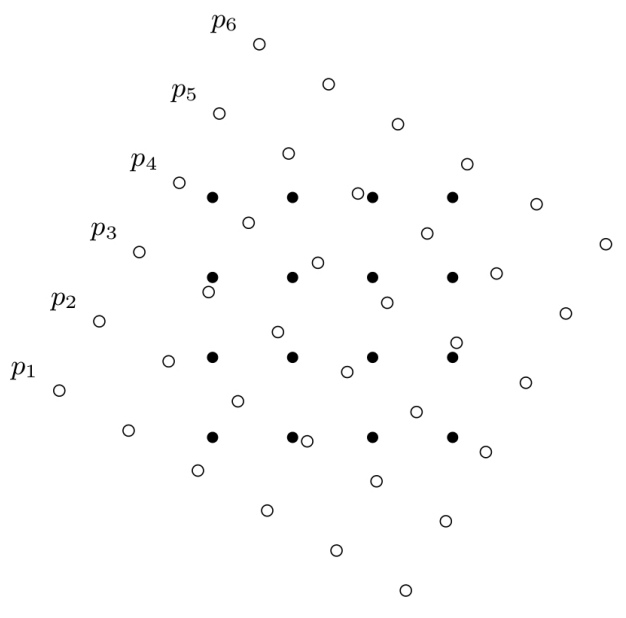

Fig. 7. Dirac representation of model on which the set up of the system of linear equations is based.

At this point it is obvious that both reconstruction techniques are not completely independent of each other as, e.g., asserted in Kak et al. (2001). Using the means of twodimensional signal processing, in particular sampling and interpolation theory Kaczmarz' method has indeed been shown to be a kind of iterative backprojection using the Radon transform to determine the estimation error. In the following Subsect. 3.2 the iterative backprojecting will be shown, for all practical means, to actually have the same transmission behaviour as the inverse Radon transform. This will also yield an explicit description of the convergence characteristics of the iterative method.

\subsection{Transmission behaviour of iterative backprojection}

In order to understand the transmission behaviour of the iterative backprojection it is self-evident to take a closer look at the Fourier space representation of Eq. (8). From Subsect. 2.2 it is known that a linear two-dimensional transmission system consisting of consecutive Radon transform and (unfiltered) backprojection can be described by an impulse response as well as a transfer function following $\frac{1}{x}$. Bearing that in mind the Fourier transform of Eq. (8) can be written as follows.

$\tilde{G}_{n+1}=\tilde{G}_{n}+\frac{1}{f_{r}} G-\frac{1}{f_{r}} \tilde{G}_{n}$

Here $f_{r}$ is the spacial frequency in radial direction and $G$ and $\tilde{G}$ are the spectra of the actual and estimated distribution functions, respectively.

The idea for deriving a closed form representation of the iterative method arises from taking a look at the first four iteration steps in Fourier space. The initial guess has been chosen according to $\tilde{G}_{0}=0$.

$\tilde{G}_{0}=0$

$\tilde{G}_{1}=\frac{1}{f_{r}} G$

$\tilde{G}_{2}=\left(\frac{2}{f_{r}}-\frac{1}{f_{r}^{2}}\right) G$

$\tilde{G}_{3}=\left(\frac{3}{f_{r}}-\frac{3}{f_{r}^{2}}+\frac{1}{f_{r}^{3}}\right) G$

$\tilde{G}_{4}=\left(\frac{4}{f_{r}}-\frac{6}{f_{r}^{2}}+\frac{4}{f_{r}^{3}}-\frac{1}{f_{r}^{4}}\right) G$

The term in brackets can be identified as part of an alternating binomial series for which the following relationship is known.

$\sum_{k=0}^{n}\left(\begin{array}{l}n \\ k\end{array}\right) a^{n-k} b^{k}=(a+b)^{n}$

This leads to the assertion that for $f_{r} \geq 1$ and $\tilde{g}_{0}=0$ the following relation holds, where $a=1$ and $b=-\frac{1}{f_{r}}$ have been used in Eq. (10).

$\tilde{G}_{n}=\left(1-\left(1-\frac{1}{f_{r}}\right)^{n}\right) G$

This can be shown by mathematical induction.

\subsubsection{Base case $(n=0)$}

$\tilde{G}_{0}=\left(1-\left(1-\frac{1}{f_{r}}\right)^{0}\right) G=0$

\subsection{Inductive step}

$$
\begin{aligned}
& (n \rightarrow n+1) \text { using (11) in (9) } \\
& \tilde{G}_{n+1}=\left(1-\left(1-\frac{1}{f_{r}}\right)^{n}\right) G+\frac{1}{f_{r}} G-\frac{1}{f_{r}}\left(1-\left(1-\frac{1}{f_{r}}\right)^{n}\right) G \\
& =\left(1-\left(1-\frac{1}{f_{r}}\right)^{n}+\frac{1}{f_{r}}\left(1-\frac{1}{f_{r}}\right)^{n}\right) G \\
& =\left(1-\left(1-\frac{1}{f_{r}}\right)\left(1-\frac{1}{f_{r}}\right)^{n}\right) G \\
& =\left(1-\left(1-\frac{1}{f_{r}}\right)^{n+1}\right) G \quad \text { Q.E.D. }
\end{aligned}
$$

(Note that the above identity can also be derived making use of the fact that Eq. (9) is a linear inhomgenous recursion formula of first order for which there is a general closed form correspondence.)

Taking the limit of Eq. (12) for $n$ to infinity clarifies the transmission behaviour of the iterative backprojection. By rewriting the term in brackets it becomes obvious that if $f_{r}$ is greater than one the bracketed term vanishes in the limit and 
the spectrum of the estimated solution $\tilde{G}_{n}$ approximates the spectrum $G$ of the actual solution with arbitrary accuracy.

$\lim _{n \rightarrow \infty} \tilde{G}_{n}=\lim _{n \rightarrow \infty}\left(1-\left(\frac{f_{r}-1}{f_{r}}\right)^{n}\right) G=G$

In the original space it then follows for the estimated and actual solutions that

$\lim _{n \rightarrow \infty} \tilde{g}_{n}=g$

Hence, for frequencies $f_{r}>1$ the iterative backprojection converges to the solution as the term in brackets vanishes in Eq. (13).

\subsection{Implications}

The first implication regards the stability of the numerical implementation of the algebraic reconstruction technique. For the numerical computation the constrain $f_{r}>1$ means that in terms of linear algebra the biggest eigenvalue of the concatenated projection and backprojection has to be lower or equal to one to guarantee convergence. In terms of the Radon and backprojection operators the operator-norm of the concatenation has to be lower or equal to one. Practically, the square sum of the pixel values, i.e. the energy of the reconstruction may not increase from one iteration step to the next. This can, e.g., be ensured by weighting the whole reconstruction with a constant factor. The closer the implementation is to the stability bound the faster the convergence.

The second implication regards the runtime of the reconstruction techniques. As for every cycle of the iterative backprojection a Radon transform and a backprojection has to be performed it is obvious that the transform-based reconstruction technique delivers its result in a fraction of the time that is needed for the algebraic reconstruction technique. Albeit, the exact time needed for the algebraic reconstruction technique depends on the required accuracy of the reconstruction which can be utilized as a criterion to abort the iteration process.

Last, the introduced relation allows to implement the algebraic reconstruction technique independently of the vectormatrix formalism avoiding the necessity to deal with handling large sparse matrices.

\section{Summary}

In this contribution the derivation of a relation between the algebraic and the transform-based reconstruction technique in computed tomography was discussed in a very general way. Therefore, based on preliminary considerations, the model for setting up the system of linear equations was reinterpreted using the means of sampling and interpolation theory which lead to understand Kaczmarz' method as iterative backprojection. The transmission behaviour of the iterative backprojection was examined and characterised offering a closed form representation of the iteration. Finally the implications and advantages of the new relation besides the academical benefit where discussed. The insights offered in this contribution give the means for further application-specific refinement and tuning of reconstruction techniques.

\section{References}

Bender, R., Gordon, R., and Herman, G.: Algebraic Reconstruction Techniques (ART) for Three-Dimensional Electron Microscopy and X-Ray Photography, in J. Theoret. Biol., 29, 471-481, 1970.

Cho, Z.-H., Jones, J. P., and Singh, M.: Foundations of medical imaging, New York: Wiley, 1993, ISBN 0-471-54573-2, 1993.

Deans, S.: The Radon transform and some of its Applications, New York, Wiley, ISBN 0-471-89804-X, 1983.

Kaczmarz, S.: Angenäherte Auflösung von Systemen linearer Gleichungen, Bull. Acad. Pol. Sci. Lett., A35, 355-357, 1937.

Kak, A., and Slaney, M.: Principles of Computerized Tomographic Imaging, New York, Society of Industrial and Applied Mathematics, ISBN 0-87942-198-3, 2001.

Morneburg, H. (Hrsg.): Bildgebende Systeme für die medizinische Diagnostik: Röntgendiagnostik und Angiographie, Computertomographie, Nuklearmedizin, Magnetresonanztomographie, Sonographie, integrierte Informationssysteme. 3., wesentlich überarb. und erw. Aufl. München: Publicis-MCD-Verl., ISBN 389578-002-2, 1995.

Poularikas, A., D. (Hrsg.): The transforms and applications handbook, Boca Raton, Fla.: CRC Press [u.a.], ISBN 0-8493-8342-0, 1996.

Radon, J.: Über die Bestimmung von Funktionen durch ihre Integralwerte längs gewisser Mannigfaltigkeiten, in: Berichte Sächsische Akademie der Wissenschaften, Math.-Phys. Kl., Leipzig, 69, 262-267, 1917.

Tanabe, K.: Projection Method for Solving a Singular System of Linear Equations and its Applications, in: Numer. Math., 17, 1971, 203-214, Springer-Verlag, 1971.

Unser, M.: Sampling-50 Years after Shannon, in: Proceedings of the IEEE, 88, 4, 569-587, Publisher Item Identifier S-00189219(00)01299-7, 2000. 